\title{
Dramatic differences in male and female timings of mortality changes for selected European cohorts for $\sim 20$ years.
}

Jeremy Clark ${ }^{1 *}$, Kamila Rydzewska ${ }^{1}$, Konrad Podsiadło ${ }^{1}$, Thierry van de Wetering ${ }^{1}$, Andrzej Ciechanowicz ${ }^{1}$

1 Department of Clinical \& Molecular Biochemistry, Pomeranian Medical University in Szczecin; ul. Powstancow Wlkp. 72, Szczecin, Zachodniopomorskie, 70-111, Poland.

*Corresponding author name and email address: Jeremy Clark, jeremyclarkbio@ gmail.com 


\section{ABSTRACT.}

Background: Longevity is of considerable interest. Collation of recent data after World War II by the Human Mortality Database allowed analyses, previously unattainable, of modal death-ages for sufficient numbers of selected European cohorts. The aim was to track modes, medians and means ( $\geq 60$ years old $(y)$ ) of all-cause mortality for both sexes. Methods: The only highestquality, large-number Lexis data available were analyzed: from nine countries: Denmark, Finland, France, Iceland, Italy, Netherlands, Norway, Sweden and Switzerland; raw-data modes (and means/medians $\geq 60 \mathrm{y}$, plus thin-plate-spline averages), were analyzed, plus pooled data. Results: Here we show that for cohorts 1880- 1900 dramatic sex differences existed between death-age changes with all countries except Iceland showing male modal negative trends lasting 10-20 years and medians in all countries near-constant or negative lasting 10-20 years; whereas females from all countries showed fairly constant positive trends (except Finnish modes and Norwegian medians). For cohorts $~ 1900-1919$ male and female modal trends were positive (except Dutch and Icelandic cohorts and Finnish females). The net results were that male mortality modes for Danish, Icelandic, Italian, Dutch, Swedish and Norwegian 1919 cohorts were roughly the same as for 1880 cohorts, whereas female death-age modes increased. Conclusion: Results clarify previously knowledge concerning sex differences during this period. Despite improved environment in late adulthood over this period, this did not translate into increased male longevity and earlier events might have sealed their fate, especially in Denmark, Italy, Netherlands, Norway, aand Sweden (and, later, Iceland).

Keywords: Cardiovascular Disease; Demography; Longevity; Thin-plate Spline. 


\section{BACKGROUND}

Longevity is of considerable interest. A lay person's view of increased longevity often means an expectation that adults have generally been living to increased ages barring early mishap (accidents and/or infectious disease), often presuming the existence of "healthy" ageing included in "life-style" mortality e.g. with cardiovascular diseases and cancer which have risk factors partly affected by life-style [1]. The question to be addresssed is whether or how this increased adult longevity has occurred for males and females over the recent past for cohorts for which data is now available i.e. for those which have recently become extinct: for example has this been continuous, intermittent or not at all for either sex?

To answer this question the choice of descriptive parameters is critical. If cohort parameters are available then the parameter "life expectancy from birth" is in general not appropriate for this purpose as Ouellette and Bourbeau [2] have indicated: "when the latest figure of life expectancy is announced, many people may take it as the "typical" (i.e. most frequent) length of life. For example, life expectancy at birth for French females is [in 2011] around 85 years. They tend to miss the fact that the typical age at death has actually been over 90 years in the period life tables since 2006." In turn, the period parameters themselves (from which life expectancies are calculated) cannot give an accurate description of what has happened to the death densities at particular times for one particular cohort; a cohort being a group born at one particular time (in this article during one year). (Period parameters come from measured deaths at one particular time for a whole population group consisting of many successive cohorts.) The choice to study cohort data in the present study, which reflect the true life histories of given birth cohorts, is therefore based on a desire to know what actually happened to particular cohorts; for 
which we now have full data (i.e. from birth to extinction), with sufficient highest-quality extinctcohort data only recently available. Note that extrapolation towards future prediction is not analyzed in the present article; note also that cohort averages are NOT available after the period studied in the present article and in the period before, which includes direct world war deaths, could be severely distorted.

It was determined to monitor death-density modes with highly-accurate cohort data from the Human Mortality Database (HMD; www.mortality.org), from all countries with Life-Table Lexis data (curated to the highest quality; these were "selected" in the sense that these were the only countries with such data): Denmark, Finland, France, Iceland, Italy, Netherlands, Norway, Sweden and Switzerland, as well as with pooled data from all countries (referred to as "Pooled", or with the largest two countries, Italy and France, removed: "EUM"). An important aspect of the work was that there were apparently no other high-quality Lexis cohort life-table data available anywhere for the period studied.

Cohorts were chosen which had sufficient data both sides of the mode to allow spline fits or to avoid World-War-II direct-death increases in mortality. Raw data, or thin-plate spline interpolation, were analyzed. Additionally, medians and means were calculated for cohort "bulk" deaths, arbitrarily defined as $\geq 60 \mathrm{y}$, using raw data or spline integration. The three most common measures of location are the mean, median and mode and all can be used to represent "typical" ages of death [3]. The benefits of using these cohort average parameters (as opposed to life expectancy) have been extensively discussed by Horiuchi et al. [3] (as well as others).

Only the major mode is independent of earlier deaths or deaths of the oldest old, and is therefore described as a robust measure of location i.e. it can be used as descriptive parameter of the death-ages of the "bulk" of adult deaths. For another thorough analysis of both period and cohort modes please see Cheung et al. [4] especially Fig 4 in this reference which shows how 
cohort major modal ages (referred to in this reference as "late modal ages" and in the present article as "modal ages") have changed for cohorts from France, Italy and Sweden.

Means and medians change according to numbers of earlier (and late) deaths and therefore a limit was arbitrarily chosen with calculation of these parameters from age 60 years old (see Kannisto on the merits or otherwise of the use of various median cut-offs [5]). (Cohen and Oppenheim [6] have also analyzed a type of median and show that these increased over the period studied for the ages birth to extinction for both males and females.) Ages above 60 years old define the "bulk" of deaths for a particular cohort in the present article (roughly corresponding to Horiuchi et al.s' "heap" in [3]).

Horiuchi et al. [3] also described the use of p-splines (as the optimal smoothing technique at that time; note that the graphs in Horiuchi et al [3] are plotted against calendar year, not cohort birth date). In the present study, in addition to the use of raw data, thin-plate splines were used to smooth death-density distributions from excessive mortality changes in particular years. Instead of using parametric methods (e.g. gompertz, logistic, Weibull, quadratic, normal or skew-t [7]) which make some assumptions concerning underlying distributions, we followed Ouellette and Bourbeau [8] and Horiuchi et al. [3] in the use of non-parametric splines. Horiuchi et al. [3] discussed the "potential theoretical importance of [the mode] in ageing research" and concluded that use of non-parametric fitting (p-splines) gave "noticeably different" modal trends to those from parametric fits (Gompertz, logistic, Weibull and their Makeham variants). Thin-plate splines can be regarded as more advanced than p-splines in the sense that they produce smooth surfaces infinitely differentiable with an interpretable energy function [9]. In addition they require no manual tuning; best fits were found automatically and visually they seemed to fit well right up to the oldest age. In contrast, with preliminary studies using p-splines the degrees of freedom had 
to be manually adjusted to achieve "best" fits (introducing questions regarding the validity of male/female comparisons if different degrees were needed for each).

A pooled analysis was also performed to give summary statistics, which might seem controversial at first sight as the countries might be expected to have very different mortality changes (see Discussion). This pooled analysis aimed to use the maximum amount of the highest quality data (from the Human Mortality Database) in order to produce overall location parameters for mortality at all ages (modes) and at ages from 60 years old (means and medians) for as many (male and female) cohorts as possible over the period studied with the maximum number of countries possible (nine countries, as in [6]). The reasoning behind this method is that the more data analyzed the smoother the density distributions become, smoothing country to country variations and providing parameters which, in any case, can be used to represent the pooled countries of Denmark, Finland, France, Iceland, Italy, Netherlands, Norway, Sweden and Switzerland.

The actual reasons for the sex differences in the timings of changes in death density distributions cannot be concluded from this study. However, some speculation can follow from previous studies. It is well established that conditions in infancy, childhood and adulthood might affect adult mortality (sometimes divided into early, middle and late life events or factors). Adult mortality is affected by earlier life events, involving factors which affect growth and development of infants, epidemiology, nutrition, salubriousness (sewerage and housing) and schooling [4], as well as access to smoking, red meat and trends in exercise, all of which affect later mortality [10]. During the period studied cardiovascular diseases gave the leading cause of world mortality with risk factors from obesity and/or smoking [11]. From this period it is possible that the "bulk" of adult deaths is increasingly determined by lifestyle, including "healthy" (if such a thing exists), mortality. The death distributions of all-cause mortality are therefore likely to 
become of great interest, especially in high-income countries, as these might start to reflect underlying biological mechanisms of aging.

It was therefore of interest to measure cohort central-tendency location parameters which describe "bulk" death-ages (i.e. from 60 years of age) without being affected by younger deaths, i.e. the modes (and medians and means above a certain age). This was possible due to recent availability of sufficient cohorts with full data from birth to extinction. The primary question investigated was: how did the differences in male and female cohort death-density distributions change over the period of study, using the highest quality data available (from the Human Mortality Database). The main a priori hypothesis was that there were changes in the modal death-ages over time in either sex in the countries analyzed.

The subject of this paper therefore concerned all-cause old-age mortality (including lifestyle mortality and/or healthy aging) in male and female cohorts born from 1880 to 1919 .

\section{METHODS and METHODOLOGY.}

\section{Data sources.}

High quality dx data was used from Cohort Life Tables from the Human Mortality Database (HMD; www.mortality.org, RRID:SCR_002370, electronic access from 2015 to 2018, last download 09-11-2018; last checked 12-08-2019), to take advantage of the decisions made by experienced demographers (methods for extracting data, and $\mathrm{dx}$ calculations, were doublechecked via email communication with HMD). Surprisingly, only few countries submit life-table cohort data, with many submitting period or other data for the study period (including e.g. USA, China). (Further high quality, large number data is apparently not available from other web sites.). Data from all available countries: Denmark, Finland, France, Iceland, Italy, Netherlands, 
Norway, Sweden and Switzerland, were analyzed separately (as well as pooled; source references are found in the Online-Resource-OR5).

For each country, all HMD cohort life-table data for one sex (Age interval×Year interval: 1x1) were downloaded into a "country" (Excel 2007; Microsoft, Washington, USA) file; plus Births data. In each country file, HMD columns were removed except: "Year" (which means year of birth in HMD cohort sections; here changed to "Cohort"), "age" and "dx"; and an additional column added: "Year of death" (="Cohort"+"age"; 110+ as 110). Separate Excel files were created for each cohort (meaning deaths of one sex born in one year), and filtered cohort data from each country file transposed into each cohort file; plus births. In a cohort file, for each country an additional row "Actual (numbers of) deaths" (dx') was calculated by [dx values $\times$ births/100000 (the radix)] (This was checked against the summed "actual deaths" for each country cohort and found to be accurate; for another use of the radix see [4]). dx' values from each country were summed to give $\mathrm{dx}^{\prime}$ values for a European pooled cohort. By duplicating these files and removing data for Italy and France the pooled data for "EUM" were created. (All cohort files are combined in "dx_primed_creation.xlsx" Online-Resources-OR1,OR2.) The pooled dx' data, plus $\mathrm{dx}^{\prime}$ data for individual countries, were transferred to the columns in "dx_primed_collation" Online-Resources-OR3,OR4.

Data from each cohort is for distinct individuals. Any (rare) missingness procedures were operated by HMD (www.mortality.org).

\section{Period of study.}

Cohorts born from 1880-1919 $(n=40)$. Cohorts were chosen with enough data so that modes of spline fits would not be affected by edge effects: the early-age and old-age borders were the ages with numbers of deaths 3/4 of numbers dying at the pooled mode (see below, and vertical lines in Fig 2). These borders were independent of the modal age; earlier cohorts were prohibited due to 
direct excess male deaths around World War II and later cohorts through lack of data. Means and medians could only be calculated for cohorts 1880 to 1904 because of lack of old age data for later cohorts. As well as the individual country and pooled analyses, the entire study was also carried out with data pooled from seven countries (without Italy and France = "EUM").

\section{Interpolation.}

Interpolation was regarded as an important way to avoid abrupt changes found in the raw data (with the assumption that people die throughout the year, not just on one particular date !) and generally spline curve smoothing was noticeable even though pooled cohorts had rather a large numbers of deaths recorded for most countries.

\section{Coding for raw data and spline analyses.}

A main coding file (Word 2007, Microsoft; Online-Resource-OR5_Coding_ INDIVIDUAL_countries) carried R coding to access the data in the Online-Resources-OR3,OR4. Analyses used the R statistical platform version 4.0 (https://cran.r-project.org). A broad plan of the coding follows. For R function stability data zeros were changed to $1 \times 10^{-2}$ and $110+\mathrm{y}$ data was kept in a separate vector. For one cohort, a thin-plate spline was fitted to the actual numbers of deaths (dx', Online-Resources-OR3,OR4) from age 40 to the oldest age (ages centered to $+0.5 \mathrm{y}$ ), using the $\mathrm{R}$ function Tps [fields] with weights $1 / \mathrm{dx}^{\prime}$ and lambda automatically found by generalized cross-validation. The function predict [fields] and $0.01 \mathrm{y}$ grids were used to determine age limits with $3 / 4$ numbers of modal deaths (two vertical lines in Fig 2). Cohorts without enough data to allow computation of these limits in the pooled analysis were not analyzed (see above). Raw modes were identified. Interpolated modal ages were found as follows. A preliminary thin-plate spline mode was found by finding the age with spline maximum (with a tie, the midway point was taken). A fine grid of interpolated age points was created around this age to find the final interpolated thin-plate spline modal age. 
Raw medians and means were calculated for each country/sex cohort from age $\geq 60$ y to 110+ (as 110) y. For each cohort the function integrate [stats], using the same age range, estimated an interpolated median by integrating successive 0.001 ages until half of the integral for the whole age range was reached, or an interpolated mean by multiplying the integrals by age values and dividing by number of values. Integrals were checked graphically with raw data. Percentages of raw numbers of death fractions (over births) were also plotted.

Non-graphical coding in two parts (for females and males) of the coding file is identical with only one part-string difference i.e. "fem" or "MALE", the female parameters computed first. Each time the main coding file was run (for a country or pooled countries) it appended 20 columns to the "parameter_results.xlsx" Online-Resource-OR6: column modeage2dp = interpolated mode; moderawage $=$ raw mode , rawMedian $=$ raw median; rawMean $=$ raw mean; IntMedian $=$ interpolated median; IntMean $=$ interpolated mean; bulkperctotal $=$ bulk deaths $($ as $\%$ of total deaths); perc76overTotal $=\%$ of fraction $>76 \mathrm{y}$; perc60to76 $=$ deaths $(\%)$ of fraction $\geq 60<76 \mathrm{y}$; perceld0 $=$ deaths $(\%)$ of fraction $\geq 95 \mathrm{y}$; with prefixes for males $(\mathrm{M})$ or females $(\mathrm{F})$ and country.

Country abbreviations used in this study: Denmark ("De" or "DEN"), Finland ("Fi", "FIN"), France ("Fr", "FRA"), Iceland ("Ic", "ICE"), Italy ("It", "ITA"), Netherlands ("Ne", "NET"), Norway ("No", "NOR"), Sweden ("Swe", "SWE" or "Sw") and Switzerland ("Swi", "SWI" or "Sz").

\section{Graphical analyses.}

Graphs of numbers of deaths against birth age for each cohort were drawn using ggplot [ggplot2] (see Figs 1:5). For individual country graphical visualisation of parameter results, loess smoothing was used with standard defaults (quadratic, span $=0.75$ ), but is only shown in supplemental figures. For all analyses residuals plots were generated. Final graphs were produced 
using graphics software (Irfanview, www.irfanview.com; Designworks version 3.5, Greenstreet Software, Huntingdon, UK).

\section{Statistics.}

Statistics coding, found in the "STATISTICS_CODING and RESULTS" Online-Resource-OR7, when run in $\mathrm{R}$ read data from Online-Resource-OR6, analyzed statistics and produced further graphs. The gradient of linear models was used for description only (not for comparison). Initially comparative linear regressions were performed but in many cases the assumptions for linear regression were not met (mostly with heteroscedasticity) and only results from Kendall independence tests are shown. For all male:female comparisons Kendall independence tests were carried out using Kendall correlation coefficients. These followed conversion from Kendall's tau to Pearson's r, as in [12], which is probably conservative. Kendall independence tests are nonparametric tests which are similar to the comparisons of slopes in analysis of covariance, but do not require the assumptions of the latter. The null hypothesis for a Kendall independence test is that the distributions are associated i.e. a significant $\mathrm{p}$ value means that the two distributions are not associated i.e. are significantly different. Male/female comparison statistical results are printed into Online-Resource-OR7, including effect sizes for linear regression and Kendall independence tests (using $\mathrm{r}=\mathrm{Z} / \sqrt{ } \mathrm{N}$ ). The alpha level for significance was set at 0.05 and all comparisons were two-tailed. Online-Resources are available for this paper and all analyses can be re-run using the coding and data files provided on (Mac; Apple Inc., Cupertino, California, USA or Windows; Microsoft, Redmond, Washington, USA) computers (further details in OnlineResource-OR5).

\section{RESULTS.}


Fig 1a shows that in all countries except Iceland death-age modes had large negative trends at some point between cohorts 1880 and $\sim 1900$ which tended to last from $\sim 10$ to $\sim 20$ years. The timings of these negative downturns varied, with Finnish and French negative trends delayed by roughly 10 years, and the Swiss male trend turning to positive at around cohort 1890 . From cohorts $~ 1900$ to 1919 the Icelandic male modal trend then turned negative (and the Netherlands at a later stage), with other countries showing positive trends. For males in Denmark, Iceland, Italy, Netherlands, Sweden and Norway the overall effect was that the modal death age at cohort 1919 was roughly the same as it was for cohort $1880(!)$.

The female trends were dramatically different, with only Finnish females showing negative trend in modal death age between cohorts 1880 and $\sim 1890$, for roughly 10 years, and female modes in all countries showing net positive increases. For cohorts $~ 1900-\sim 1919$ female modal trends in some countries (Netherlands, Iceland and Finland) showed negative trends but in Denmark, France, Italy, Norway, Sweden and Switzerland were positive. All female cohorts showed a net overall increase in modal death age between cohorts 1880 and 1919.

The thin-plate spline interpolated modes (Fig 1b) showed similar trends with the sex differences slightly clearer. (It should be noted that the timings as shown by the thin-plate splines could be regarded as more reliable.)

\section{FIGURE 1}

The pooled data (in which the trends are naturally weighted by the number of individuals) provide simple statistical summaries to the above results. Typical pooled mortality density curves using numbers of deaths estimated from $\mathrm{dx}$ data $\left(\mathrm{dx}^{\prime}\right)$ for male and female cohorts born in 1881 common era year (CE) are shown in Fig 2. Fig 2 a shows the bump at $\sim 65 \mathrm{y}$ from direct male 
World-War-II deaths which deterred use of cohorts before 1880 CE. (Narrow confidence intervals are not shown as these would be hardly visible but can be generated by running OnlineResource-OR5.) Pooled modal death ages are shown in Fig 3 and pooled data without the largest countries France and Italy are shown in Supplemental-Figs-S1:S3.) All summary statistical graphs simply present the data in a different way but with weighting, and the conclusions drawn are similar to those from individual country graphs (Figs 1 and 4).

\section{FIGURE 2}

\section{FIGURE 3}

Fig 4 shows that in Sweden, Italy, Norway, the Netherlands and Denmark median or mean deathages were either near-constant or showed an increase followed by a protracted decrease over the 25 years that medians could be calculated. Icelandic males also showed a decrease after 1890 . In France, Switzerland and Finland these parameters increased (although the medians were nearconstant for approximately 10 years during this period, see Fig 4b).

In all countries female bulk medians and means showed increases over 25 years (apart from in Norway during the first 5 years).

Fig 5 shows the overall summary statistics for how pooled raw cohort means and medians ( $\geq 60$ y) changed over time: male medians constant at $76 \mathrm{y}$, whereas means increased slightly; female averages increased dramatically over this 25 year period. (Integrated median/mean graphs are similar, see Supplemental-FigS4; and results are also similar if Italian and French data are removed: see Supplemental-Figs-S1:S3) Note the summary statistical graphs simply provide another way of showing the data (but are naturally weighted according to numbers of individuals). 


\section{FIGURE 4}

\section{FIGURE 5}

Kendall independence tests showed significant differences between sexes $(\mathrm{p}<0.001)$ for all pooled comparisons (see Figs 3,5) showing that these had moved differently over time. These tests can also be generated for all individual countries by running OR5_Coding_INDIVIDUAL_countries in R. Note the Kendall independence tests show whether the male and female changes were associated or not. (Loess-smoothed curves can also be generated, i.e. equivalent to Figs 1 and 4, for all analyses by running Online-Resource-OR5 in R; the loess standard errors are very small for both males and females, presumably because of the very large datasets, in these graphs.)

Further statistics including effect sizes and confidence intervals for Kendall independence tests are given in Online-Resource-OR7. If Online-Resource-OR7 is run in $\mathrm{R}$ then results and graphs can be obtained and, in addition, results are also printed into this file. All residuals plots were generated but slight curvatures in the data do not affect the non-parametric statistical comparisons.

\section{DISCUSSION.}

\section{Individual country analyses.}

The individual country results (Fig 1) are the most important and show dramatic sex differences in mortality changes over time. In all countries, male modal averages showed negative trends for at least $\sim 10$ years during the study period and for Denmark, Iceland, Italy, Netherlands, Sweden and Norway the end result was that the modal death age at cohort 1919 was roughly the same as 
at cohort 1880. In contrast female modal death-ages (Fig 4b) were higher at the end of the study period in all countries. In Fig 1 in Horiuchi et al. [3] similar results can be seen for French males and females (note that in this reference these are plotted against calendar year, not cohort birth date); and other studies have previously presented modal death-ages [13, 14]. Beltrán-Sánchez et al. [11] analyzed cohorts with birth dates between 1800 to 1935 in 13 countries and stated that "a relatively new demographic phenomenon .... emerged among people born in the late 19th century", referring to the divergence.

Divergence between male and female modal death-ages in particular countries during the study period has been previously noted by many studies e.g. see [4] (and Fig 7 in this reference; with quadratic fits) in which French and Swedish data are shown for this period (note the x-axis in this Figure shows calendar year, not cohort birth date, and the study concerns mostly period comparisons).

Male medians and means (Fig 4) showed, over the 25 years for which they could be calculated, that Danish, Dutch, Italian, Norwegian and Swedish 1904 cohort values were similar or less than for cohort 1880. In these five countries males showed decreasing or near-constant median death-ages over this period (with a hump with Netherland medians resulting in an overall decrease). Net median and mean changes for Finland, France, Iceland and Switzerland were positive, although note that the medians for these countries were near-constant for a considerable period ( 10 years). It is clear from Fig 4 a that the male median death-ages in all countries did not change in accordance with female medians, which all showed rapid increases (with Norway female median increase delayed by around 5 years). (Integrated results, shown in SupplementalFigS4, were similar to the raw results shown in Fig 4.)

It must be remembered that means are highly sensitive to changes in the death-ages of the oldest old and should therefore be treated with caution in their use as location parameters for 
"bulk" deaths. Technically conclusions regarding modes are independent of other distributional characteristics whereas the medians depend on the lower cut-off boundary chosen: here $\geq 60 \mathrm{y}$, but are also more robust than the means. Conclusions are therefore mostly drawn from the modes which cover 40 years of the study and, to a lesser extent, the medians, which cover the first 25 years.

\section{Thin-plate splines.}

Thin-plate splines are not necessary to show the main effects which can be seen quite clearly using raw data. However, the modal results from thin-plate spline fits not only showed reduced standard errors around the loess lines, but also the timings of some modal changes were noticeably different between raw data and thin-plate spline fits: compare Fig 1a and 1b; for example, the peak for male Icelandic modes is at 1898 for raw data, but $\sim 1902$ for thin-plate splines, and the male lines for Netherlands and Denmark differ. It is expected that p-splines would also give different timings but not necessarily exactly as for thin-plate splines, and the latter could be regarded as optimal. The robustness of the thin-plate spline fits can be seen visually by looking closely at fits to the oldest old up to $110 \mathrm{y}$ in the cohort graphs in Fig 2 and in those seen if the main coding is run from Online-Resource-OR5: the fits appear to be good in every case, despite the low amounts of data at the oldest ages.

It might be argued that the smoothing of the modes provided by the thin-plate splines is too great but this is not the case. This can be seen from Fig $3 a$ in which the male raw modal death ages (Fig 3a) give iterated downward slopes which correspond to peaks resulting from particular years with excessive mortality which have affected several cohorts at once at different ages. That these have been correctly smoothed can be seen by running the main coding (Online-ResourceOR5: the graphs will run like a motion picture film) in which such peaks will be seen to move from right to left while the thin-plate spline mode is more stable. 


\section{Study limitations.}

(1) As Crimmins et al. [15] have indicated, conclusions might well only apply to the countries studied. For many large countries e.g. the USA or China, life-table Lexis data were not available for the period studied, and caution is necessary to draw conclusions from other data types e.g. period data. We could not add data similar to that analyzed because, as far as we know, none exists. (2) The nine countries contributed $\sim 30 \%$ of the non-Russian/non-Ottoman European population [16]; pooled conclusions do not necessarily apply to Europe as a whole, and are only given as summary statistics. (3) Although conclusions regarding modes are independent of population fraction, medians and means are affected by the fraction analyzed (here $\geq 60 \mathrm{y}$ ) and medians and means would change if another fraction were considered.

\section{Health, stress and mortality.}

It is important to realise that we do not know the reasons as to why male average death-ages showed decreases (modes) or were usually near-constant (other averages), in contrast to female parameters, over the study period. In a recently published and authoritative article concerning health, morbidity and mortality, Crimmins et al. [15] stated that "our strongest conclusion is that male/female differences in health are highly dependent on historical time and geographic location." This is certainly true for the countries analyzed and we sincerely hope that downward trends in male mortality parameters are quirks due to historical events which will not be repeated, but this cannot be guaranteed.

It is possible that downward trends in male mortality were predominantly affected by early or middle-age factors and not late-age factors, or that there were serious male-biased factors which off-set positive late-age factors, for two reasons: (1) during the period in which these males were actually dying (at least after World War II), there has been a fairly constant increase in gross domestic product per person, health care, diet and general living standards in all the 
countries studied, which suggests that mortality in these males was either not affected by these improvements or there were factors which seriously offset these; (2) the female average deathages increasesd during the same period, and presumably females were exposed to many of the same factors. As we find it unlikely that late-age factors do not have effects on male mortality, we concentrate on male-biased off-sets, which might themselves have originated in early, middle or late-age. Even so, the results appear to provide weak evidence that early or middle-age factors, rather than late-age factors, are critical for male mortality.

Current thinking is, as mentioned in the Introduction, that as mortality due to infectious diseases decreased, then cardiovascular disease, cancers and other chronic diseases became more important factors influencing mortality [15]. Mortality, however, has multivarious causes, and factors affecting infancy, adolescence and young adulthood might well have more influence than is at first apparent. According to Cohen and Oppenheim [6] mortality depends on environmental, behavioural, sanitary, nutritional, and medical conditions as well as public health, social, political and economic organisation, food supplies, education and human values.

Possibilities for the divergence in male/female mortality rates from 1880 onwards might have arisen from:

(A) a greater vulnerability of males to cardiovascular diseases and a higher frequency of lethal conditions such as heart disease, stroke, and diabetes [15]. Perhaps most importantly for 50-70 year olds from the cohorts studied, dramatic sex differences were found in UK cardiovascular disease deaths which began $\sim 1925$, with male numbers increasing to $\sim 1975$, but female numbers decreased (see Fig 1 in [17]), and similar trends were found in Europe [18]. Quite possibly this increase in male-cardiovascular and other life-style disease frequencies contributed to the male longevity stagnation and after this period there was a measured fall in e.g. cardiovascular 
diseases, which might explain why the male modal death ages in most countries subsequently increased.

The genetic basis as to why men might be more susceptible to chronic diseases such as cardiovascular diseases is well known, with strong sexual dimorphism in aging and survival disadvantage among men likely resulting from a male-specific mitochondrial mutation load, which might also affect (B) and (C) below [15].

(B) different behavioural responses to environment, including male increased uptake of smoking [15] (relative to women), and with increased wealth an increase in access to red meat, alcohol and less overall exercise, which all increased the risk of cardiovascular diseases. It is thought that men are "more likely to engage in risky and dangerous behavior and women more likely to engage in health-seeking behavior" $[15,19]$. Animal product consumption has been suggested to have significantly contributed to cardiovascular and/or cancerous diseases, and there are hints that they may have greater negative effects in men than women [20]. Possibly males did not (or were unable to) take advantage of beneficial environmental changes. We can speculate that as prosperity increased, women thrived with better life-style choices, whereas males might have relatively consumed more red meat and alcohol, smoked, and become less active: all risk factors for an earlier death [21].

(C) greater susceptibility of males to the long term effects of the 1918 influenza pandemic (colloquially known as the "Spanish Flu"). This hypothesis cannot be discounted as a major determinant yet because of the huge numbers of people infected (it caused acute illness in 25-30 percent of the world's population [22]) plus the fact that the sex differences in infection rates were enormous with an age-standardized death-rate difference of 174 per $100 \quad 000$ [23]. According to Azambuja [24] white men born from 1880 to 1900 (20-40 years old) were 
preferentially killed because of an unusual immune response and survivors of infection might also have exhibited the same response giving a "primed" later susceptibility to coronary heart disease mortality [24].

While (C) is an interesting theory, and one which could be further investigated by comparing the timing of age-distributional infection rates in individual countries with the graphs in Figs $1 \mathrm{~b}$ and Supplemental-FigS4, it should be noted that recently some evidence has been gathered against this idea [25]. In this reference a small study compared $>1000$ military males who had contracted 1918 influenza with controls and found no differences in long-term survival [25], but as the authors mention this is probably not applicable to whole populations including non-military-personnel. As the world is currently in the midst of a Covid-19 pandemic, lessons learned from this period might be directly relevant to those living today.

(D) Although explanations concerning cardiovascular diseases seem persuasive, in any case for the generation studied many had fought in two wars and the deferred effects of war (psychological/injury) might have exacerbated behavioral or other differences. It may be that the cohorts studied show peculiar features with atypical mortality patterns which might reflect, for instance, harmful effects from the two world wars. Such effects can be seen among cohorts which directly participated in a war, or in people who during adolescence suffered from undernourishment due to a war. However, it must be remembered that war-related hypotheses need to account for differences (or lack of them) between countries at war or neutral countries: in particular note that Sweden and Switzerland were neutral throughout both world wars but have different male trends (see Fig 4), and France and Italy were at war in both world wars but also have different trends (see Figs 1 and 4). Denmark, The Netherlands and Norway were neutral throughout World War I, and have overall decreases in average death-ages during this period, 
which might count against a war-related hypothesis (unless war had positive effects on later mortality).

(E) Excess male migration is also a factor which might be considered (with predominantly healthy males migrating).

Any theory which purports to explain the presented results will need to explain why the scourge which affected males did so at different times in different countries. It is therefore interesting to note that with some cardiovascular risk markers at some times and in some countries, women have have had higher or similar cardiovascular risk to that of males [15]. As Crimmins et al. [15] have indicated, hypertension levels (a risk factor for cardiovascular diseases) are usually greater for males than females in most countries, but there are several countries where the prevalence of hypertension is higher for women.

At some time after the study period sex differences in mortality changes likely become much less apparent. According to Crimmins et al. [15] "sex differences in disease prevalence and mortality rates may recede" as risks for cardiovascular disease mortality reduce and as men and women behave more similarly. For example, data from the National Health and Nutrition Examination Survey (NHANES) showed that by 2010 there were no sex differences in mean agespecific cardiovascular risk markers at ages $>50$ years [26], and in the USA and Europe there has been increasing similarity in smoking habits between men and women [27].

The use of optimal parameters will be critical in the analysis of the timing of past events. Of some importance could be the fact that the timing, of e.g. changes in modal death-ages, sometimes depends on whether thin-plate splines have been used or not; the thin-plate splines theoretically providing a more accurate representation. In the future, as cohort parameter data catch up with data collected on health dimensions (which have only been collected since the 1980s with national-level surveys on multiple dimensions of morbidity for large samples of both 
sexes $[1,15])$ it will be the cohort parameters with optimal smoothing functions (e.g. thin-plate splines) which will provide the correct insights into the timing of past events. With the present study it might be possible to resolve factors by investigation of relative timings, e.g. of the 1918 influenza pandemic, in different countries, especially for example in comparison with Iceland.

We hope that the way in which the data has been presented (which we think is optimal) will encourage further research into these mortality changes - especially relative to the timings of the many factors possibly associated with mortality.

\section{Notes on pooled analyses.}

It is important to note that the main results and conclusions do NOT depend on the pooled results, which are simply statistical summaries with natural weighting according to the numbers of individuals. It is easy to see from Figs 1 and 4 that the male averages proceeded roughly from left to right and the female averages proceeded roughly upwards in a slanting direction showing increases in average death ages. However, it is nice to have summary statistical graphs (Figs 2, 3 and 5) as these show the trends rather clearly, and the following should be noted:

1) It might seem strange to pool the results from large and disparate countries such as Italy and France, but it must be remembereed that the large and disparate cities of Milan and Naples have already been pooled in the Italian data, and likewise for Paris and Marseille in France. At present we have no idea whether the overall timings in male negative trends differ or are similar between these four cities. Italy and France contributed approximately one third each to the numbers, but if these countries are removed from the pooled analyses (see Supplemental-Figs-S1:S3) the shapes of the curves are almost the same, showing that these distribution shapes are robust. 
2) All the male data show negative modal trends over some periods (due to unknown causes), and all male median data show periods of near-constancy, and therefore have some similarity.

3) There might be some reluctance to analyse individual country data further, on the grounds that it is not known whether differences in the average parameters are bounded by country borders, or whether, for example, they vary from city to city, plus the fact that some countries, e.g. Iceland, have considerably less data and are therefore more influenced by random fluctuations in regulating factors. The pooled data from nine countries represent the sum total of highest-quality human data knowledge concerning the mortality density of these cohort bulks and the large datasets have smooth density distributions (even without splines, see Fig 2), which is less apparent with individual country data.

4) Even if you don't accept points (1) to (3) it still remains that these are valid statistical summaries.

\section{Other types of study.}

It might also be tempting to compare the results obtained with those of life expectancy at birth, but the latter is inappropriate to answer the question raised if cohort parameters are available, and this has not been done (see Introduction; similar arguments apply to life expectancy from age 60 y). As Cheung et al. [4] have stated: "the advantages of using the late modal age at death ......[have been] regularly underlined in the past [28-30]". The study of "rectangularization" [31] or compression of mortality [4] could also be studied, although this phenomenon appears to be waning $[6,32]$.

The present study has used some of the best techniques and only the highest quality data available to calculate cohort death-density modes using raw data or thin-plate splines. Other parameters could be used e.g. mortality rates, but we think dx' gives clarity. 


\section{Conclusions.}

In summary, for cohorts 1880- 1900 dramatic sex differences existed between death-age changes with all countries except Iceland showing male modal negative trends lasting 10-20 years and medians in all countries near-constant or with negative trends lasting $~ 10-20$ years; whereas females from all countries showed fairly constant positive trends (except Finnish modes and Norwegian medians). For cohorts $\sim 1900-1919$ male and female modal trends were positive (except Dutch and Icelandic cohorts and Finnish females). The net results were that male mortality modes for Danish, Icelandic, Italian, Dutch, Swedish and Norwegian 1919 cohorts were roughly the same as for 1880 cohorts, whereas female death-age modes had net increases. There are many possible causes for the sex differences in the timings of mortality changes for cohorts born during this period. Suspicion lies with changes connected with cardiovascular and other "life-style" diseases. It is a sobering thought that at least a part of a generation of men might in general not have taken (or been able to take) advantage of environmental changes for the opportunity for longer life and/or might have suffered the long-term effects of the 1918 influenza pandemic and/or the deferred effects of war.

\section{List of Abbreviations.}

CE, common era year; fem, female; dx, density; dx', actual numbers of deaths estimated from density (dx) data; EUM, refers to pooled data from all countries except Italy and France: Denmark, Finland, Iceland, Netherlands, Norway, Sweden and Switzerland; HMD, Human Mortality Database; NHANES, National Health and Nutrition Examination Survey; S.D., standard deviation; USA, United States of America; Column names in Online-Resource-OR6: modeage $2 \mathrm{dp}=$ interpolated mode $;$ moderawage $=$ raw mode; rawMedian $=$ raw median; 
rawMean = raw mean; IntMedian = interpolated median; IntMean = interpolated mean; bulkperctotal $=$ bulk deaths $($ as $\%$ of total deaths); perc76overTotal $=\%$ of fraction $>76 \mathrm{y}$; perc60to76 $=$ deaths $(\%)$ of fraction $\geq 60<76 \mathrm{y}$; perceld $0=$ deaths $(\%)$ of fraction $\geq 95 \mathrm{y}$; with prefixes for males (M) or females (F) and country: Denmark ("De" or "DEN"), Finland ("Fi", "FIN"), France ("Fr", "FRA"), Iceland ("Ic", "ICE"), Italy ("It", "ITA"), Netherlands ("Ne", "NET"), Norway ("No", "NOR"), Sweden ("Swe", "SWE") and Switzerland ("Swi", "SWI").

\section{Declarations.}

Funding. This study was funded entirely by the Pomeranian Medical University, Szczecin, Poland. Conflicts of interest/Competing interests. The authors have no conflicts of interest to declare that are relevant to the content of this article. Ethics approval. Not applicable. Consent to participate. Not applicable. Consent for publication. Not applicable. Availability of data and material. All data, code and all calculations can be found in the Online Resources and the Human Mortality Database (www.mortality.org). Code availability. All data and all calculations can be found in the Online Resources. All residual plots can be generated using Online Resource coding. Correspondence and requests for materials should be addressed to JSCC. Authors' contributions: JSCC contributed to concept/design, data analysis/interpretation, statistical analysis, drafting the article; KR, KP, TW to data analysis/interpretation, statistical analyses, critical revision of article, TW, AC to critical revision of article. Acknowledgements. We would like to thank the Human Mortality Database staff for their help and availability of data on their website at www.mortality.org. 


\section{References}

1. Seeman TE, Singer BH, Rowe JW, Horwitz RI, McEwen BS. Price of adaptation-allostatic load and its health consequences. MacArthur studies of successful aging. Archives of Internal Medicine. 1997; 157:2259-2268.

2. Ouellette N, Bourbeau R. Changes in the age-at-death distribution in four low mortality countries: A nonparametric approach. Demographic Research. 2011;25:595-628.

3. Horiuchi S, Ouellette N, Cheung SLK, Robine J-M. Modal age at death: lifespan indicator in the era of longevity extension. Vienna Yearbook of Population Research. 2013;11:37-69.

4. Cheung SLK, Robine JM, Caselli G. The use of cohort and period data to explore changes in adult longevity in low mortality countries. Genus. 2008;64:101-129.

5. Kannisto V. Mode and Dispersion of the Length of Life. Population. 2001;13:159-171.

6. Cohen JE, Oppenheim J. Is a limit to the median length of human life imminent? Genus. 2012;68:11-40.

7. Clark JSC, Kaczmarczyk M, Mongiało Z, Ignaczak P, Czajkowski AA, Klęsk P, Ciechanowicz A. Skew-t Fits to Mortality Data-Can a Gaussian-Related Distribution Replace the Gompertz-Makeham as the Basis for Mortality Studies? The Journals of Gerontology: Series A. 2013;68:903-913.

8. Nadine O, Robert B. Changes in the age-at-death distribution in four low mortality countries: A nonparametric approach. Demographic Research. 2011;25:595-628.

9. Nychka D, Furrer R, Paige J, Sain S. Fields: Tools for spatial data. Boulder, CO, USA: University Corporation for Atmospheric Research; 2017

10. Robine J-M. Life Course, Environmental Change, and Life Span. Population and Development Review. 2003;29:229-238.

11. Beltrán-Sánchez H, Soneji S, Crimmins EM. Past, Present, and Future of Healthy Life Expectancy. Cold Spring Harbor Perspectives in Medicine. 2015;5

12. Walker D. JMASM9: Converting Kendall's Tau For Correlational Or Meta-Analytic Analyses. Journal of Modern Applied Statistical Methods. 2003;2

13. editor. A bio-actuarial approach to forecasting rates of mortality. Proceedings of the Centerary Assembly of the Institute of Actuaries. 2; 1950; 1950.

14. Le Bras H. Lois de mortalité et age limite. Population (French Edition). 1976;31:655-692.

15. Crimmins EM, Shim H, Zhang YS, Kim JK. Differences between Men and Women in Mortality and the Health Dimensions of the Morbidity Process. Clinical Chemistry. 2019;65:135-145.

16. Wood J. The Nuttall Encyclopaedia. London and New York: Frederick Warne.; 1920

17. Nikiforov SV, Mamaev VB. The development of sex differences in cardiovascular disease mortality: a historical perspective. American Journal of Public Health. 1998;88:1348-1353.

18. Glei DA, Horiuchi S. The narrowing sex differential in life expectancy in high-income populations: effects of differences in the age pattern of mortality. Population Studies. 2007;61:141-159.

19. Rogers RG, Everett BG, Saint Onge JM, Krueger PM. Social, Behavioral, and Biological Factors, and Sex Differences in Mortality. Demography. 2010;47:555-578. 
20. Montesanto A, De Rango F, Pirazzini C, Guidarelli G, Domma F, Franceschi C, Passarino G. Demographic, genetic and phenotypic characteristics of centenarians in Italy: Focus on gender differences. Mechanisms of Ageing and Development. 2017;165:68-74.

21. Zheng Y, Li Y, Satija A, Pan A, Sotos-Prieto M, Rimm E, Willett WC, Hu FB. Association of changes in red meat consumption with total and cause specific mortality among US women and men: two prospective cohort studies. BMJ. 2019;365

22. Taubenberger JK. The Origin and Virulence of the 1918 "Spanish" Influenza Virus. Proceedings of the American Philosophical Society. 2006;150:86-112.

23. Noymer A, Garenne M. The 1918 Influenza Epidemic's Effects on Sex Differentials in Mortality in the United States. Population and development review. 2000;26:565-581.

24. Azambuja MIR. Spanish Flu and Early 20th-Century Expansion of a Coronary Heart Disease-Prone Subpopulation. Texas Heart Institute Journal. 2004;31:14-21.

25. Wilson N, Boyd M, Nisa S, Clement C, Baker MG. Did exposure to a severe outbreak of pandemic influenza in 1918 impact on long-term survival? Epidemiology $\backslash \&$ Infection. 2016;144:3166-3169.

26. Kim JK, Ailshire JA, Crimmins EM. Twenty-year trends in cardiovascular risk among men and women in the United States. Aging Clinical and Experimental Research. 2019;31:135143.

27. Janssen F. Similarities and Differences Between Sexes and Countries in the Mortality Imprint of the Smoking Epidemic in 34 Low-Mortality Countries, 1950-2014. Nicotine $1 \&$ Tobacco Research. 2020;22:1210-1220.

28. Elderton WP. Graduation and Analysis of a Sickness Table. Biometrika. 1903;2:260-272.

29. Greenwood M, Irwin JO. The biostatistics of senility. Human Biology. 1939;11:1-23.

30. Gumbel EJ. La Duree Extreme de la Vie Humaine. Hermann and Co; 1937

31. Fries JF. Aging, natural death, and the compression of morbidity. The New England Journal of Medicine. 1980;303:130-135.

32. Wilmoth JR, Horiuchi S. Rectangularization revisited: Variability of age at death within human populations. Demography. 1999;36:475-495.

Data source references (required by the Human Mortality Database rules) are found at the end of Online-Resource-OR5. 


\section{Figure legends.}

Fig. 1 Modal death ages: Individual countries. Female and male cohort modal death ages (years old; y; loess-smoothed) from (a) raw mortality (dx') data or (b) interpolated thin-plate splines; from individual countries, versus cohort birth date (common era year; CE) or date cohort defined as extinct (at 110 y). Females (blue dot-dashed lines; italic labels); Males (red or black lines, upright labels): De, Denmark; Fi, Finland; Fr, France; Ic, Iceland; It, Italy; Ne, Netherlands; No, Norway; Sw, Sweden; Sz, Switzerland. Female modes increased, but in five (red solid line) countries, male modes gave negative net modal death age over first 30 years. Source of raw data: Human Mortality Database (2019)

Fig. 2 Death $\left(d^{\prime}\right)$ distribution summary statistics, 1881 CE. Numbers of deaths (estimated from $\mathrm{dx}$ ) from male $(\mathrm{a}, \mathrm{x})$ and female $(\mathrm{b},+)$ pooled cohorts from 1881 common era year (CE) versus age of death (years old; y). Curves show thin-plate spline fits (narrow confidence intervals not shown but can be generated by running Online-Resource-OR5). Vertical lines show ages with interpolated numbers of deaths $3 / 4$ of numbers dying at the mode. The second-world-war directdeath mortality bump is seen in the male, but not female, cohort. Source of raw data: Human Mortality Database (2019)

Fig. 3 Modal death ages summary statistics. Female (+; solid line: 30 year trend) and male (x; dashed line) cohort modal death ages (years old; y) from (a) raw mortality (dx') data; or (b) interpolated thin-plate splines; versus cohort birth date (common era year; CE) or date cohort 
defined as extinct (at $110 \mathrm{y}$ ). Interpolated pooled modal-death ages show a near-constant increase with female, but not male, cohorts. Source of raw data: Human Mortality Database (2019)

Fig. 4 Cohort (a) median, and (b) mean, death ages: Individual countries. Female and male cohort loess-smoothed (a) median or (b) mean death ages (years old; y) from individual-country raw mortality (dx') data, versus cohort birth date (common era year; CE) or date cohort defined as extinct. Females (blue dot-dashed lines; italic labels); Males (red or black lines, upright labels): De, Denmark; Fi, Finland; Fr, France; Ic, Iceland; It, Italy; Ne, Netherlands; No, Norway; Sw, Sweden; Sz, Switzerland. For both averages, female death-ages increased but in five (red solid) countries male death-ages had net decrease or were near-constant over the first 30 years. Source of raw data: Human Mortality Database (2019)

Fig. 5 Cohort (a) median, and (b) mean, death ages. Female (+; dashed line) and male (x; solid line) cohort (a) median or (b) mean death ages (years old; y) from raw mortality (dx') data, versus cohort birth date (common era year; CE) or date cohort defined as extinct (at $110 \mathrm{y}$ ). Raw male median-death ages are exactly constant over this period whereas females' increase. Source of raw data: Human Mortality Database (2019)

\section{Online Resources:}

OR1_Female_dx_primed_creation.xlsx

Pooling calculations for Pooled Female data from nine countries.

\section{OR2_Male_dx_primed_creation.xlsx}

Pooling calculations for Pooled Male data from nine countries.

\section{OR3_Female_dx_primed_collation.xlsx}


Collated $\mathrm{dx}^{\prime}$ female data from Pooled data, EUM (= with Italy and France removed) and individual countries.

\section{OR4_MALE_dx_primed_collation.xlsx}

Collated dx' male data from Pooled data, EUM (= with Italy and France removed) and individual countries.

\section{OR5_Coding_INDIVIDUAL_countries.docx}

Main coding file. If all header rows are removed (apart from one) from OR3 and OR4 and these saved as .csv, when OR5 is run in $\mathrm{R}$ it will read OR3 and OR4 and produce results for chosen country, or for Pooled data or EUM, which will be written to OR6.

\section{OR6_parameter_results.xlsx}

Results from running OR5.

\section{OR7_STATISTICS_CODING and RESULTS.docx}

Statistics coding file. If all header rows are removed (apart from one) from OR6 and this saved as .csv, when OR7 is run in R it will read OR6 and generate statistics and graphs. Results have been printed into OR7.

\section{Supplemental Figure legends.}

Supplemental-FigS1 Death $\left(\mathrm{dx}^{\prime}\right)$ distribution summary statistics for seven countries, 1881.

Data pooled from all countries except France and Italy ("EUM"). Numbers of deaths (estimated from $\mathrm{dx}$ ) from male $(\mathrm{a}, \mathrm{x})$ and female $(\mathrm{b},+)$ pooled cohorts from 1881 common era year (CE) versus age of death (years old; y). Curves show thin-plate spline fits (narrow confidence intervals not shown but can be generated by running Online-Resource-OR5). Vertical lines show ages with interpolated numbers of deaths $3 / 4$ of numbers dying at the mode. In contrast to the pooled study with nine countries, the second-world-war direct-death mortality bump can hardly be seen at all with the male data. Source of raw data: Human Mortality Database (2019) 


\section{Supplemental-FigS2 Modal death age summary statistics for seven countries ("EUM").}

Female (+; solid line: 30 year trend) and male (x; dashed line) cohort modal death ages (years old; y) from (a) raw mortality (dx’) data; or (b) interpolated thin-plate splines; versus cohort birth date (common era year; CE) or date cohort defined as extinct (at $110 \mathrm{y}$ ). Interpolated pooled modal-death ages show a near-constant increase with female, but not male, cohorts. Source of raw data: Human Mortality Database (2019)

\section{Supplemental-FigS3 Cohort (a) median and (b) mean summary statistics for severn} countries ("EUM"). Female (+; dashed line) and male (x; solid line) cohort (a) median or (b) mean death ages (years old; y) from raw mortality (dx') data, versus cohort birth date (common era year; CE) or date cohort defined as extinct (at 110 y). Raw male median-death ages show a slight decline over this period whereas females' increase; raw male mean-death ages are almost constant. Source of raw data: Human Mortality Database (2019)

\section{Supplemental-FigS4 Cohort (a) median, and (b) mean, interpolated death ages for}

individual countries. Female and male cohort loess-smoothed (a) median or (b) mean death ages (years old; y) from individual-country integrated thin-plate spline fits to mortality (dx') data, versus cohort birth date (common era year; CE) or date cohort defined as extinct. Females (blue dot-dashed lines; italic labels); Males (red or black lines, upright labels): De, Denmark; Fi, Finland; Fr, France; Ic, Iceland; It, Italy; Ne, Netherlands; No, Norway; Sw, Sweden; Sz, Switzerland. Standard errors (grey). For both averages, female death-ages increased but five (red solid) countries male death-ages showed net decreases or were near-constant over the first 30 years. Source of raw data: Human Mortality Database (2019) 
bioRxiv preprint doi: https://doi.org/10.1101/2020.05.23.111971; this version posted May 3, 2021. The copyright holder for this preprint (which was not certified by peer review) is the author/funder, who has granted bioRxiv a license to display the preprint in perpetuity. It is made available under aCC-BY-NC 4.0 International license. 


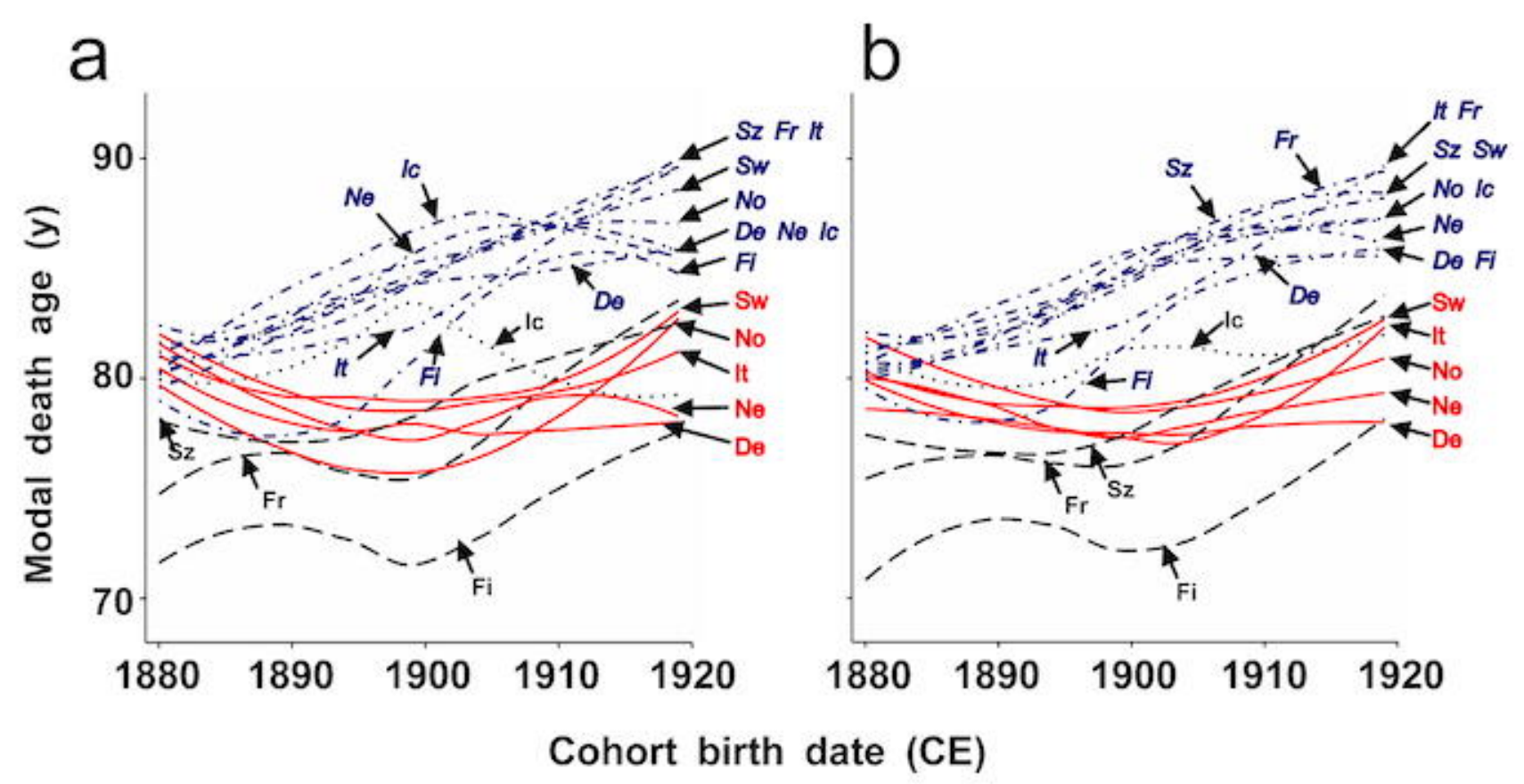

\begin{tabular}{|c|c|c|c|c|c|c|c|c|c|}
\hline 1990 & 2000 & 2010 & 2020 & 2030 & 1990 & 2000 & 2010 & 2020 & 2030 \\
\hline
\end{tabular}




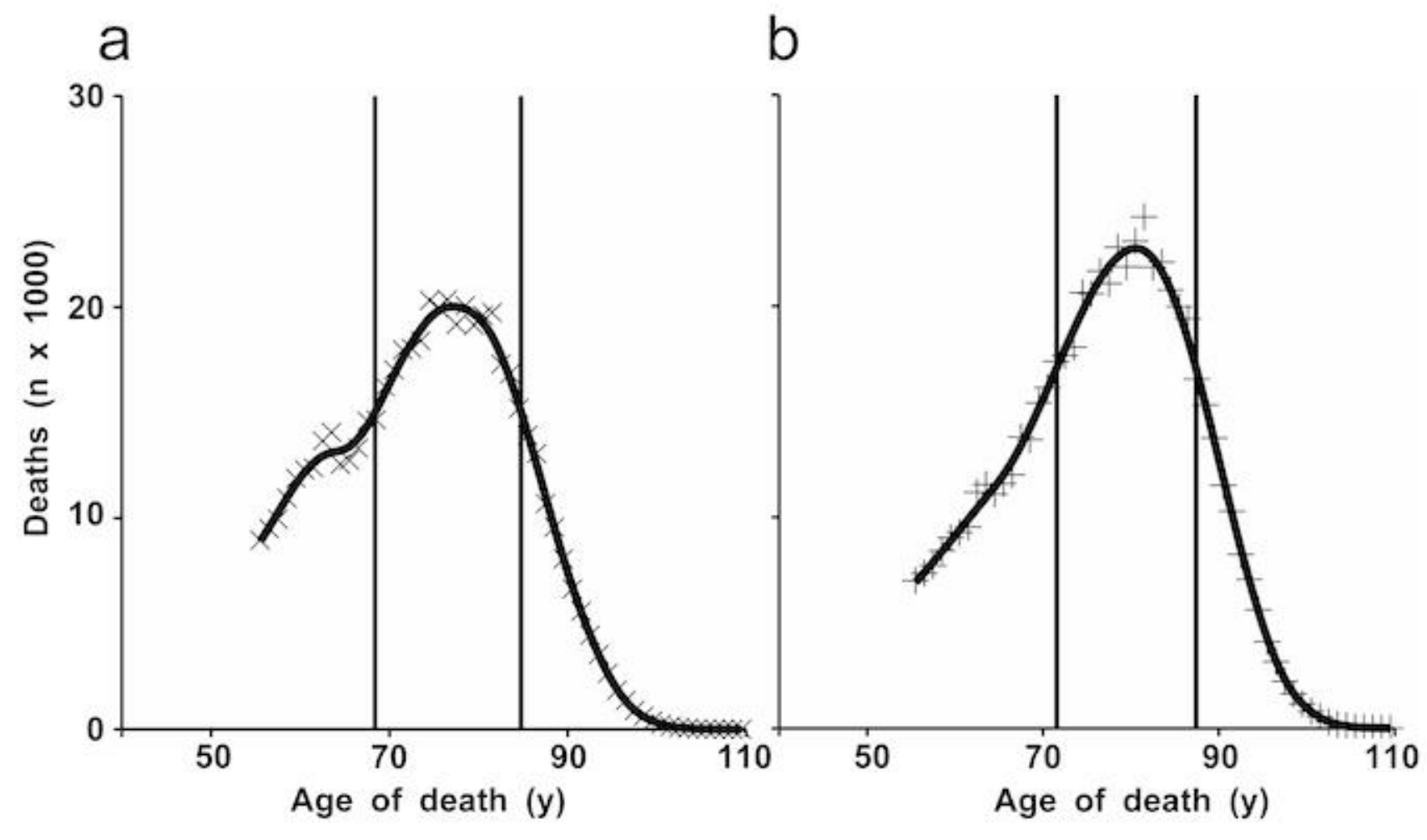


a b

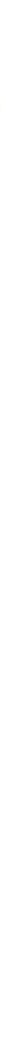




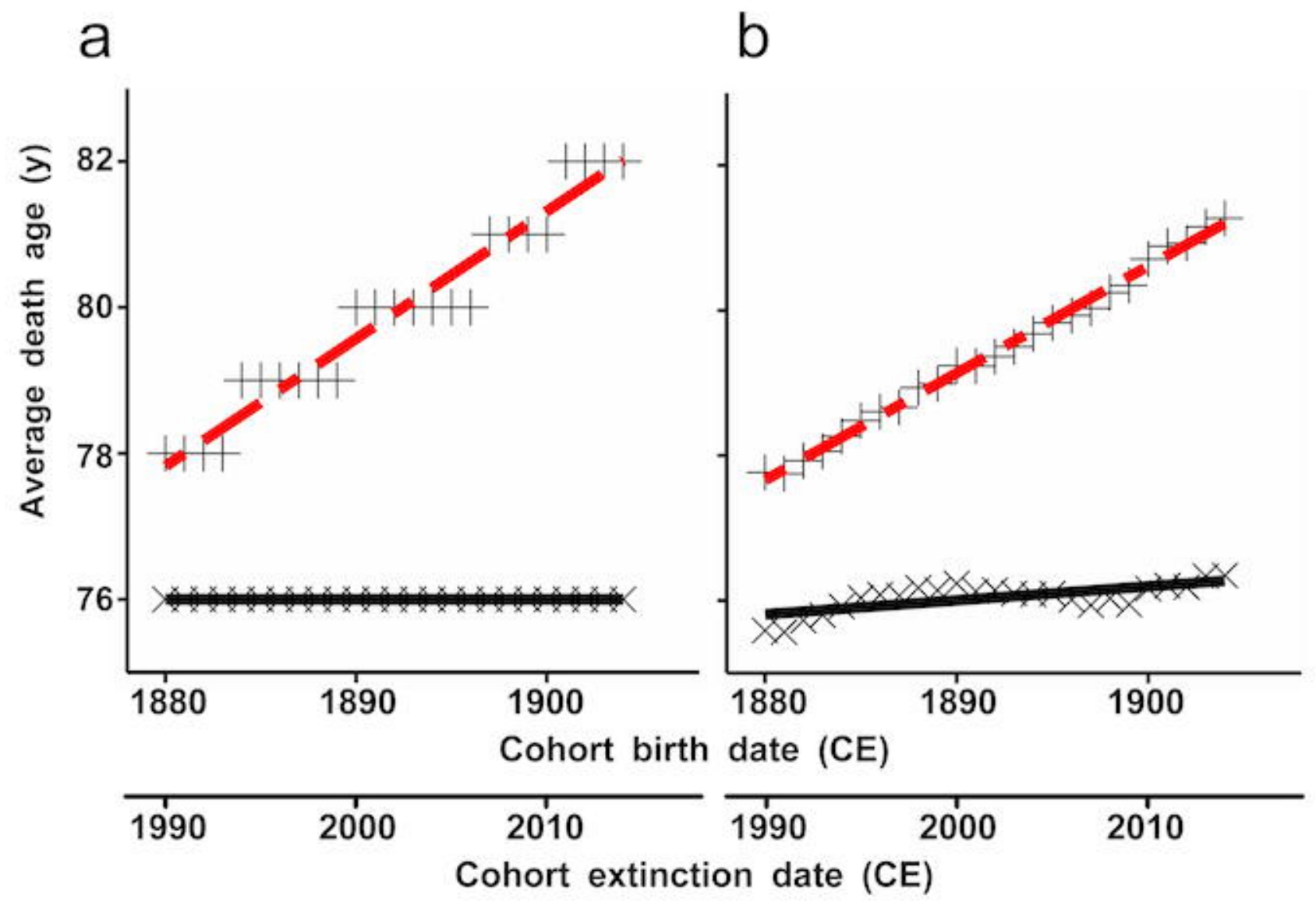

